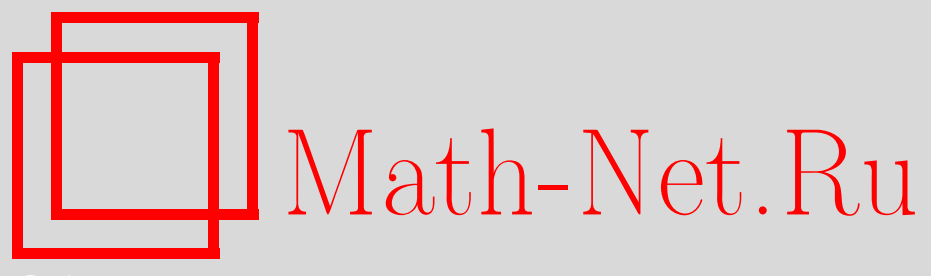

В. В. Белов, Ф. Н. Литвинец, А. Ю. Трифонов, Квазиклассические спектральные серии оператора типа Хартри, отвечающие точке покоя классической системы Гамильтона-Эренфеста, ТМФ, 2007, том 150, номер 1, 26-40

DOI: https://doi.org/10.4213/tmf5964

Использование Общероссийского математического портала Math-Net.Ru подразумевает, что вы прочитали и согласны с пользовательским соглашением http: //www . mathnet.ru/rus/agreement

Параметры загрузки:

IP: 52.90 .164 .192

26 апреля 2023 г., 16:12:21

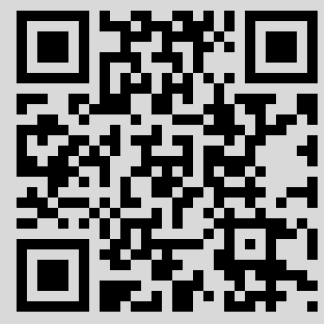




\title{
КВАЗИКЛАССИЧЕСКИЕ СПЕКТРАЛЬНЫЕ СЕРИИ ОПЕРАТОРА ТИПА ХАРТРИ, ОТВЕЧАЮЩИЕ ТОЧКЕ ПОКОЯ КЛАССИЧЕСКОЙ СИСТЕМЫ ГАМИЛЬТОНА-ЭРЕНФЕСТА
}

\begin{abstract}
Рассмотрены классические уравнения движения относительно квантовых средних - система Гамильтона-Эренфеста. В рамках основанного на этих уравнениях ковариантного подхода в квазиклассическом приближении построены спектральные серии для нелинейного оператора типа Хартри, отвечающие точке покоя.
\end{abstract}

Ключевые слова: метод комплексного ростка, спектральные серии, уравнение Хартри.

\section{1. ВВЕДЕНИЕ}

Предметом исследования статьи является спектральная задача для нелинейного оператора Хартри в квазиклассическом приближении

$$
\widehat{H}_{\varkappa} \Psi=E \Psi, \quad\|\Psi\|_{L_{2}\left(\mathbb{R}^{n}\right)}=1,
$$

где самосопряженный в $L_{2}\left(\mathbb{R}^{n}\right)$ оператор типа Хартри действует по формулам

$$
\begin{aligned}
& \widehat{H}_{\varkappa} \Psi=H(\hat{z}) \Psi+\varkappa \widehat{V}(\Psi) \Psi, \\
& \widehat{V}(\Psi)=\int_{\mathbb{R}^{n}} d y \Psi^{*}(y) V(\hat{z}, \widehat{w}) \Psi(y) .
\end{aligned}
$$

Здесь $\hat{z}=\left(-i \hbar \nabla_{x}, x\right), \widehat{w}=\left(-i \hbar \nabla_{y}, y\right), x, y \in \mathbb{R}^{n}$, самосопряженные в $L_{2}\left(\mathbb{R}^{n}\right)$ операторы $H(\hat{z}), V(\hat{z}, \widehat{w})$ являются гладкими функциями от некоммутирующих операторов и упорядочены по Вейлю [1], [2], $\Psi^{*}$ - комплексно сопряженная к $\Psi$ функция, $\varkappa$ и $\hbar$ - вещественные параметры, $\hbar \in[0,1)$ - "малый" параметр. Важным является

* Московский институт электроники и математики, Москва, Россия.

E-mail: pm@miem.edu.ru

${ }^{\dagger}$ Томский политехнический университет, Томск, Россия.

E-mail: litvinets@mph.phtd.tpu.edu.ru, trifonov@phtd.tpu.edu.ru 
частный случай уравнения (1), когда вейлевские символы операторов в $(2),(3)$ имеют вид $H(z)=p^{2} / 2+U(x), p \in \mathbb{R}^{n}, V(z, w)=V(x, y)$, и уравнение (1) записывается в виде

$$
\left[-\frac{\hbar^{2}}{2} \Delta+U(x)+\varkappa \int_{\mathbb{R}^{n}} V(x, y)|\Psi(y)|^{2} d y\right] \Psi=E \Psi .
$$

Это уравнение самосогласованного поля во внешнем поле с потенциалом $U(x)$ и потенциалом самодействия $V(x, y)$ играет фундаментальную роль в квантовой теории и нелинейной оптике [3], в частности, в теории бозе-эйнштейновского конденсата [4] и при описании коллективных возбуждений в молекулярных цепочках и в молекулах ДНК [5].

В линейной теории (когда в $(1) \varkappa=0$ ) аппарат квазиклассических асимптотик, возникший практически одновременно с квантовой механикой, позволяет обосновать важный для физической теории принцип соответствия [6] квантовых и классических задач. В определенном смысле математическое обоснование принципа соответствия дает теория Маслова [7], охватывающая наряду со спектральными задачами задачу с начальными данными и задачу рассеяния (см. также [8]).

Одним из важнейших моментов квазиклассического приближения является сведе́ние построения квазиклассического решения квантовой задачи к исследованию решений уравнений движения соответствующей классической системы, ее геометрических и топологических характеристик. Для “линейной" квантовой механики $(\varkappa=0$ в (1)) соответствующая классическая система - это априори гамильтонова система в фазовом пространстве $\mathbb{R}^{2 n}$ с канонической 2-формой $d p \wedge d x$ и гамильтонианом $H(z), z=(p, x),-$ символом оператора $H(\hat{z})$. В качестве геометрических объектов фазового пространства, порождающих квазиклассические ответы, выступают изотропные подмногообразия $\Lambda^{k},\left.(d p \wedge d x)\right|_{\Lambda^{k}}=0$, размерности $k, 0 \leqslant k \leqslant n$, где $n$ - размерность конфигурационного пространства.

В спектральных задачах квазиклассические собственные функции и собственные значения (спектральные серии, или квазимодъ [9], [10]) можно сопоставить компактным $\Lambda^{k}$ лишь при условии их инвариантности относительно фазового потока $g_{H}^{t}\left(\Lambda^{k}=g_{H}^{t} \Lambda^{k}\right)$. При этом в маломерном случае, т.е. при $k<n$, инвариантные изотропные подмногообразия должны удовлетворять дополнительному условию типа условия устойчивости. На геометрическом языке это означает существование инвариантного комплексного ростка Маслова $r^{n}$ над $\Lambda^{k}$ [10], образованного комплексными траекториями линеаризованной системы в окрестности $\Lambda^{k}$. В простейшем случае $k=0$, когда $\Lambda^{0}$ - устойчивая точка покоя гамильтонова векторного поля $J \nabla_{z} H(z)$, метод построения соответствующих квазимод на основе комплексного ростка (канонического оператора Маслова с комплексной квадратичной фазой [9], [10]), разумеется, эквивалентен известному в физике методу осцилляторного приближения в окрестности точки $\Lambda^{0}$. В случае замкнутой фазовой кривой $\Lambda^{1}(k=1)$ условие орбитальной устойчивости $\Lambda^{1}$ (условие существования комплексного ростка $r^{n}$ над $\left.\Lambda^{1}\right)$ эквивалентно существованию базиса Флоке решений соответствующей системы в вариациях, косоортогональных касательному вектору к кривой $\Lambda^{1}[10]$.

Обобщение рассмотренных конструкций на случай нелинейных квантовых систем заведомо нетривиально, поскольку сама постановка задачи о соответствии "классике" уже является проблематичной, так как неясно (в отличие от линейного случая), 
что понимать под уравнениями классической механики, отвечающей в пределе $\hbar \rightarrow 0$ заданной квантовой теории с нелинейным гамильтонианом $\widehat{H}_{\varkappa}(2)$.

Ответ на этот вопрос зависит, по видимому, от класса функций, в котором строятся асимптотические разложения решения уравнения (1), и от свойств гладкости символов оператора $\widehat{H}_{\varkappa}$. Так, например, для уравнения (4) с сингулярным потенциалом самодействия $V(x, y)$ кулоновского типа квазимоды, сосредоточенные при $\hbar \rightarrow 0$ вблизи маломерных подмногообразий в $\mathbb{R}^{n}$, были получены в [11] на основе "сингулярного" варианта метода ВКБ с помощью эталонных уравнений.

Для общего оператора типа Хартри (2) с гладкими символами $H(z), V(z, w)$ удалось в рамках ковариантного подхода, развитого в цикле работ [12]-[15] вывести классические уравнения движения, определяющие квазиклассические асимптотики с точностью $O\left(\hbar^{3 / 2}\right)$ (далее будем писать $\left.\left(\bmod \hbar^{3 / 2}\right)\right)$ нестационарной задачи

$$
\begin{gathered}
i \hbar \Psi_{t}=\widehat{H}_{\varkappa} \Psi, \quad\|\Psi\|_{L_{2}\left(\mathbb{R}^{n}\right)}=1, \\
\left.\Psi\right|_{t=0}=\Psi_{0}(x, \hbar)
\end{gathered}
$$

в так называемом классе функций $P_{\hbar}^{t}$, квазиклассически сосредоточенных при $\hbar \rightarrow 0$ на заданной кривой $x=X(t, \hbar) \in \mathbb{R}_{x}^{n}$, регулярно зависящей от малого параметpa $\hbar[16]$.

Напомним определение указанного выше класса функций (см., например, [12]). Пусть $Z(t, \hbar)=(P(t, \hbar), X(t, \hbar))$ - произвольное гладкое однопараметрическое $(\hbar-$ параметр) семейство фазовых траекторий в $\mathbb{R}^{2 n}$. Тогда

$$
P_{\hbar}^{t}=\left\{\Phi, \Phi=\phi\left(\frac{x-X(t, \hbar)}{\sqrt{\hbar}}, t\right) \exp \left[\frac{i}{\hbar}(S(t, \hbar)+\langle P(t, \hbar), x-X(t, \hbar)\rangle)\right]\right\},
$$

где $S(t, \hbar)$ - вещественная гладкая по $t$ и $\hbar$ функция, $S(0, \hbar)=0$, и функция $\phi(\xi, t)$ по переменной $\xi \in \mathbb{R}^{n}$ принадлежит пространству Шварца $S\left(\mathbb{R}^{n}\right)$.

Как показано в [12], [13], асимптотические $\left(\bmod \hbar^{3 / 2}\right)$ решения задачи $(5)$ определяются решениями системы обыкновенных дифференциальных уравнений относительно динамических переменных $z=(p, x) \in \mathbb{R}^{2 n}$ и переменных $\eta=\left(\eta_{1}, \ldots, \eta_{k}, \ldots\right.$ $\left.\ldots, \eta_{M}\right) \in \mathbb{R}^{M}, M=n(2 n+1)$, смысл которых будет пояснен ниже. Набор этих переменных $\eta$ удобно представить в виде блочной $(2 n \times 2 n)$-симметричной матрицы

$$
\Delta=\left(\begin{array}{cc}
\Delta_{1} & \Delta_{2} \\
\Delta_{3} & \Delta_{4}
\end{array}\right), \quad \Delta_{1}^{\mathrm{T}}=\Delta_{1}, \quad \Delta_{4}^{\mathrm{T}}=\Delta_{4}, \quad \Delta_{2}^{\mathrm{T}}=\Delta_{3}
$$

где $A^{\mathrm{T}}$ - матрица, транспонированная к $A$. Определим в расширенном фазовом пространстве $\mathbb{R}^{2 n} \times \mathbb{R}^{2 n} \times \mathbb{R}^{M}$ функцию Гамильтона с самодействием

$$
H^{\varkappa}(z, w, \Delta)=H(z)+\varkappa V(z, w, t)+\frac{1}{2} \operatorname{Sp}\left[\left(H_{z z}^{\prime \prime}(z)+\varkappa V_{z z}^{\prime \prime}(z, w)+\varkappa V_{w w}^{\prime \prime}(z, w)\right) \Delta\right] .
$$

Здесь через $H_{z z}^{\prime \prime}(z)$ и $V_{w w}^{\prime \prime}(z, w)$ обозначены $(2 n \times 2 n)$-матрицы вторых производных функций $H(z)$ и $V(z, w)$ по переменным $z=(p, x)$ и $w=\left(p_{y}, y\right)$. Определим симметричную $(2 n \times 2 n)$-матрицу

$$
M_{\varkappa}(z)=\frac{\partial^{2}}{\partial z^{2}} H^{\varkappa}(z, z, 0)=H_{z z}(z)+\left.\varkappa V_{z z}(z, w)\right|_{w=z} .
$$


Тогда классическая система, отвечающая оператору Хартри $\widehat{H}_{\varkappa}(2)$ и названная в [12], [13] системой Гамильтона-Эренфеста, имеет вид

$$
\begin{gathered}
\dot{z}=\left.J \nabla_{z} H^{\varkappa}(z, w, \Delta)\right|_{w=z}, \\
\dot{\Delta}=J M_{\varkappa}(z) \Delta-\Delta M_{\varkappa}(z) J, \quad \Delta^{\mathrm{T}}=\Delta .
\end{gathered}
$$

Здесь $J$ - стандартная симплектическая матрица, $J=\left(\begin{array}{cc}0 & -E \\ E & 0\end{array}\right), \quad E=\left\|\delta_{i j}\right\|_{n \times n}$.

ЗАмЕчАниЕ. В линейном случае (при $\varkappa=0$ ) система $(11)$ является пуассоновой системой относительно (вырожденной) нелинейной скобки Дирака [17]. При $\varkappa \neq 0$ вопрос о свойствах гамильтоновости системы (11) требует дополнительного исследования.

Физическое "обоснование" того, что систему (11) можно считать классической системой, отвечающей квантовой системе с гамильтонианом $\widehat{H}_{\varkappa}(2)$, основано на следующей концепции, лежащей в основе ковариантного подхода [18] (эта концепция обобщает первоначальный подход Эренфеста [6] к проблеме вывода классических уравнений движения в приближении $\hbar \rightarrow 0$ из уравнения Шредингера). Под фазовой классической траекторией в $\mathbb{R}^{2 n}$ квантовой частицы, находящейся в состоянии $\Psi(x, t, \hbar)$, где $\Psi(x, t, \hbar)$ - решение уравнения $(5)$, понимается вектор-функция $z^{\Psi}(t, \hbar)=(P(t, \hbar), X(t, \hbar))$, компоненты которой суть средние значения операторов координат и импульсов в состоянии $\Psi: P(t, \hbar)=\langle\Psi,-i \hbar \nabla \Psi\rangle, X(t, \hbar)=\langle\Psi, x \Psi\rangle$, $x \in \mathbb{R}^{n}$. Здесь $\langle\cdot, \cdot\rangle$ - скалярное произведение в $L_{2}\left(\mathbb{R}^{n}\right)$. Если приближенное или точное решение $\Psi(x, t, \hbar) \in P_{\hbar}^{t}$, то в системе Гамильтона-Эренфеста (11) дополнительные по отношению к $(p, x) \in \mathbb{R}^{2 n}$ динамические переменные $\eta_{k}, k=1, \ldots, M$, учитывают в первом приближении по $\hbar \rightarrow 0$ квадратичные квантовые флуктуации координат и импульсов около их предельных значений $X(t, 0), P(t, 0)$. В частности, начальные данные Коши $\Psi_{0} \in P_{\hbar}^{0}$ для уравнения (5) индуцируют следующие начальные условия для системы (11):

$$
\begin{gathered}
\left.x\right|_{t=0}=x_{0}(\hbar)=\left\langle\Psi_{0}, x \Psi_{0}\right\rangle,\left.\quad p\right|_{t=0}=p_{0}(\hbar)=\left\langle\Psi_{0},-i \hbar \nabla \Psi_{0}\right\rangle, \\
\left.\Delta\right|_{t=0}=\Delta\left(\Psi_{0}\right),
\end{gathered}
$$

где $\Delta\left(\Psi_{0}\right)$ - матрица квантовых центрированных моментов второго порядка для операторов $(-i \hbar \nabla), x \in \mathbb{R}^{n}$, в состоянии $\Psi_{0}$. Это означает, что $(n \times n)$-блоки матрицы $\left.\Delta\right|_{t=0}$ имеют вид

$$
\Delta_{4}=\sigma_{x x}\left(\Psi_{0}\right), \quad \Delta_{1}=\sigma_{p p}\left(\Psi_{0}\right), \quad \Delta_{2}=\sigma_{p x}\left(\Psi_{0}\right), \quad \Delta_{3}=\sigma_{p x}^{\mathrm{T}}\left(\Psi_{0}\right),
$$

где

$$
\sigma_{A B}=\frac{1}{2}\langle\Delta \hat{A} \Delta \widehat{B}+\Delta \widehat{B} \Delta \hat{A}\rangle, \quad \Delta \hat{A}=\hat{A}-\langle\hat{A}\rangle, \quad \Delta \widehat{B}=\widehat{B}-\langle\widehat{B}\rangle .
$$

Через $\langle\hat{A}\rangle$ обозначено среднее значение оператора $\hat{A}$ с символом $A$ в состоянии $\Psi_{0}:\langle\hat{A}\rangle=\left\langle\Psi_{0}|\hat{A}| \Psi_{0}\right\rangle$.

Нетрудно установить (см. [16]), что на функциях из класса $P_{\hbar}^{0}$ матрица $\Delta\left(\Psi_{0}\right)$ имеет порядок $O(\hbar), \hbar \rightarrow 0$. Таким образом, решения классической системы (11), 
определяющие квазиклассическую асимптотику решения задачи (5), сами, в свою очередь, зависят регулярным образом от малого параметра $\hbar$ квазиклассического приближения в квантовой задаче. Этот факт имеет принципиальное значение для построения квазимод оператора типа Хартри (2) в рамках ковариантного подхода.

Классическая система Гамильтона-Эренфеста (11) позволяет теперь определить (как и в линейном случае) однопараметрическое семейство $\Lambda^{k}(\hbar), 0 \leqslant k \leqslant n$, инвариантных геометрических объектов в фазовом пространстве $\mathbb{R}^{2 n} \times \mathbb{R}^{M}$, которые можно проквантовать в квазиклассическом приближении с точностью до $O\left(\hbar^{3 / 2}\right)$, т.е. сопоставить им спектральные серии задачи $(1)-$ числа $E_{\nu}$ и функции $\Psi_{\nu}(x, \hbar)$ такие, что $\widehat{H}_{\varkappa} \Psi_{\nu}=E_{\nu} \Psi_{\nu}+O\left(\hbar^{3 / 2}\right), \hbar \rightarrow 0,\left\|\Psi_{\nu}\right\|_{L_{2}\left(\mathbb{R}^{n}\right)}=1, \nu$ - мультииндекс длины $n$. В простейших случаях это точки покоя $\Lambda^{0}(\hbar)$ системы (11) или ее замкнутые траектории $\Lambda^{1}(\hbar)$.

Цель этой работы - квазиклассическое квантование точек покоя системы Гамильтона-Эренфеста, ассоциированной с нелинейным гамильтонианом $\widehat{H}_{\varkappa}$, при следующих условиях на вейлевские символы операторов $H(z), V(z, w)$ в $(2),(3)$ : функции $H(z)$ и $V(z, w)$ являются $\mathbb{C}^{\infty}$-гладкими функциями и вместе со своими производными по $z$ и $w$ растут при $|z| \rightarrow \infty$ и $|w| \rightarrow \infty$ не быстрее, чем полином, т.е. для любых мультииндексов $\alpha, \mu \in \mathbb{Z}_{+}^{2 n}$ существуют постоянные $C_{\alpha}>0, C_{\alpha \mu}>0$ и $m>0$ такие, что выполняются неравенства

$$
\left|\frac{\partial^{|\alpha|} H(z)}{\partial z^{\alpha}}\right| \leqslant C_{\alpha}(1+|z|)^{m}, \quad\left|\frac{\partial^{|\alpha+\mu|} V(z, w)}{\partial z^{\alpha} \partial w^{\mu}}\right| \leqslant C_{\alpha \mu}(1+|z|)^{m}(1+|w|)^{m} .
$$

Здесь

$$
\begin{gathered}
\alpha=\left(\alpha_{1}, \alpha_{2}, \ldots, \alpha_{2 n}\right), \quad \alpha_{j} \geqslant 0, \quad|\alpha|=\alpha_{1}+\alpha_{2}+\cdots+\alpha_{2 n}, \quad \alpha !=\alpha_{1} ! \alpha_{2} ! \ldots \alpha_{n} !, \\
z^{\alpha}=z_{1}^{\alpha_{1}} z_{2}^{\alpha_{2}} \ldots z_{2 n}^{\alpha_{2 n}}, \quad \frac{\partial^{|\alpha|} V(z)}{\partial z^{\alpha}}=\frac{\partial^{|\alpha|} V(z)}{\partial z_{1}^{\alpha_{1}} \partial z_{2}^{\alpha_{2}} \ldots \partial z_{2 n}^{\alpha_{2 n}}} .
\end{gathered}
$$

Основная идея построения соответствующих квазимод заключается в том, чтобы найти асимптотическое решение $\Psi(x, t, \hbar) \in P_{\hbar}^{t}$ задачи Коши $(5)$, зависящее от времени по гармоническому закону: $\Psi(x, t, \hbar)=\exp [i \lambda t / \hbar] \Psi_{\lambda}(x, \hbar)$. Ясно, что тогда пара $\left(\lambda, \Psi_{\lambda}\right)$ есть квазимода оператора $\widehat{H}_{\varkappa}$.

Оказывается, что это можно сделать, если использовать так называемые траекторно-когерентные состояния нестационарного уравнения типа Хартри [12], [13]. Они отвечают специальному выбору амплитуды в начальном условии (6). А именно, в качестве $\phi_{0}\left(\frac{x-x_{0}}{\sqrt{\hbar}}\right)$ следует выбрать счетный набор фоковских состояний $\Phi_{\nu}$, $\nu \in \mathbb{Z}_{n}^{+}$, многомерного осциллятора [19], параметризованных комплексной симметричной матрицей с положительной мнимой частью. В разделе 2 мы приводим, следуя общей схеме из [12], [13], алгоритм построения соответствующего $(2 n+M)$ параметрического семейства $\Psi_{\nu}(x, \hbar)$ таких решений. Этот алгоритм при каждом фиксированном $\nu$ сопоставляет решению $y_{\nu}\left(t, \Psi_{0}\right)=\left(P(t, \hbar), X(t, \hbar), \Delta_{\nu}(t, \hbar)\right)$ задачи Коши для системы Гамильтона-Эренфеста (11)-(13) (при $\Psi_{0}=\exp \left[\frac{i}{\hbar}\left\langle p_{0},(x-\right.\right.$ $\left.\left.\left.x_{0}\right)\right\rangle\right] \Phi_{\nu}$ в $\left.(6)\right)$ асимптотические $\left(\bmod \hbar^{3 / 2}\right)$ решения, которые мы обозначим здесь через $\Psi\left(x, t, \hbar, y_{\nu}\left(t, \Psi_{0}\right)\right)=\Psi_{\nu}(x, t)$. Параметры семейства этих решений однозначно определяются (см. раздел 3 ) из требования, чтобы решение $y_{\nu}\left(t, \Psi_{0}\right)$ было стационарным, т.е. являлось точкой покоя системы Гамильтона-Эренфеста. В этом случае 
$\Psi_{\nu}(x, t)$ - циклические по времени решения. Оказывается, что существование точек покоя системы (11) эквивалентно устойчивости системы в вариациях с самодействием, возникающей в ковариантном подходе, и это представляет собой аналог условия устойчивости точек покоя гамильтоновой системы в линейной теории. В разделе 3 мы формулируем основной результат работы и приводим явные формулы соответствующих квазимод, а в разделе 4 рассматриваем ряд примеров, иллюстрирующих эти формулы.

\section{2. КОНСТРУКЦИЯ ТРАЕКТОРНО-КОГЕРЕНТНЫХ СОСТОЯНИЙ НЕСТАЦИОНАРНОГО УРАВНЕНИЯ ТИПА ХАРТРИ}

Вначале опишем счетный ортонормированный набор начальных данных $\Psi_{\nu}(x, \hbar)$ $\in P_{\hbar}^{0}\left(\nu-\right.$ мультииндекс, $\left.\nu \in \mathbb{Z}_{+}^{n}\right)$ для уравнения (5), порождающих асимптотические $\left(\bmod \hbar^{3 / 2}\right)$ решения $\Psi_{\nu}(x, t, \hbar) \in P_{\hbar}^{t}$, которые мы называем траекторнокогерентными состояниями нелинейного уравнения типа Хартри по аналогии с линейной теорией [16]. Для линейных квантовомеханических уравнений (Шредингера, Клейна-Гордона, Паули и Дирака-Паули) траекторно-когерентные состояния были построены в работах [20].

Пусть фиксированы точка $\left(p_{0}, x_{0}\right) \in \mathbb{R}^{2 n}$ и две комплексные матрицы $B_{0}, C_{0}$, удовлетворяющие условиям

$$
B_{0}^{\mathrm{T}} C_{0}-C_{0}^{\mathrm{T}} B_{0}=0, \quad B_{0}^{+} C_{0}-C_{0}^{+} B_{0}=2 i E, \quad E=\left\|\delta_{i j}\right\|_{n \times n} .
$$

Определим набор функций $\Psi_{\nu}(x, \hbar)=\Psi_{\nu}\left(x, \hbar, x_{0}, p_{0}, B_{0}, C_{0}\right), \quad \nu \in \mathbb{Z}_{n}^{+}$, зависящий от параметров $x_{0}, p_{0}, B_{0}, C_{0}$, формулами

$$
\begin{aligned}
& \Psi_{\nu}(x, \hbar)=N_{\nu} \exp \left[\frac{i}{\hbar}\left\langle p_{0}, x-x_{0}\right\rangle\right] \Phi_{\nu}(x, \hbar), \\
& \Phi_{\nu}(x, \hbar)=\exp \left[\frac{i}{2 \hbar}\left\langle x-x_{0}, B_{0} C_{0}^{-1}\left(x-x_{0}\right)\right\rangle\right] \frac{1}{\sqrt{\operatorname{det} C_{0}}}\left[\widehat{\Lambda}^{+}(0)\right]^{\nu} 1 .
\end{aligned}
$$

Здесь $N_{\nu}$ - нормировочная постоянная, $N_{\nu}=(\sqrt{2})^{-|\nu|}(\pi \hbar)^{-n / 4} / \sqrt{\nu !},\left[\widehat{\Lambda}^{+}(0)\right]^{\nu}=$ $\left[\widehat{\Lambda}_{1}^{+}\right]^{\nu_{1}}\left[\widehat{\Lambda}_{2}^{+}\right]^{\nu_{2}} \ldots\left[\widehat{\Lambda}_{n}^{+}\right]^{\nu_{n}}$, где $\widehat{\Lambda}_{j}^{+}, j=1,2, \ldots, n,-$ обобщенные операторы рождения (см. [16]), отвечающие матрицам $B_{0}, C_{0}$,

$$
\widehat{\Lambda}_{j}^{+}=\frac{1}{\sqrt{\hbar}}\left(\left\langle z_{j}^{*}, \hat{p}\right\rangle-\left\langle w_{j}^{*}-B_{0} C_{0}^{-1} z_{j}^{*}, x-x_{0}\right\rangle\right),
$$

а $z_{j}, w_{j}, j=1,2, \ldots, n,-$ вектор-столбцы матриц $C_{0}$ и $B_{0}$, соответственно. Звездочка над вектором означает комплексное сопряжение. Нетрудно проверить, что в силу свойств (15) матрица $B_{0} C_{0}^{-1}$ существует, симметрична и имеет положительную мнимую часть $\operatorname{Im} B_{0} C_{0}^{-1}=\left(C_{0}^{+} C_{0}\right)^{-1}$. При этом операторы $\widehat{\Lambda}_{j}^{+}$попарно коммутируют друг с другом, и, следовательно, действие оператора $\widehat{\Lambda}^{+}$на 1 не зависит от порядка действия компонент вектор-оператора $\widehat{\Lambda}^{+}(0)=\left(\widehat{\Lambda}_{1}^{+}, \widehat{\Lambda}_{2}^{+}, \ldots, \widehat{\Lambda}_{n}^{+}\right)$.

Отметим, что набор функций $\Psi_{\nu}, \nu \in \mathbb{Z}_{n}^{+}$, также как и фоковский базис функций $\Phi_{\nu}, \nu \in \mathbb{Z}_{n}^{+}$, образует ортонормированный базис в пространстве $L_{2}\left(\mathbb{R}^{n}\right)[16]$, причем, $\left[\widehat{\Lambda}^{+}(0)\right]^{\nu} 1$ есть операторное представление многомерных полиномов Эрмита $H_{\nu}(\xi)$, 
порожденных матрицей $W_{0}=-C_{0}^{+}\left(C_{0}^{-1}\right)^{\mathrm{T}}[21]$. Здесь $\xi=-i \sqrt{2 / \hbar} C_{0}^{-1} \Delta x, \quad \Delta x=$ $x-x_{0}$.

Как уже отмечалось во введении, квазиклассические асимптотики задачи (5), (6) однозначно определяются по решениям соответствующей системы Гамильтона-Эренфеста. Обозначим через $y\left(t, \Psi_{\nu}(0)\right)=(P(t, \hbar), X(t, \hbar), \Delta(t, \hbar)) \in \mathbb{R}^{2 n} \times \mathbb{R}^{M}$ гладкое однопараметрическое семейство решений системы (11) на интервале $[0, T]$ с начальными данными (12), (13), в которых $p_{0}(\hbar)=p_{0}, x_{0}(\hbar)=x_{0}$, а матрица дисперсий $\Delta\left(\Psi_{0}\right)$, вычисленная по состояниям $\Psi_{0}=\Psi_{\nu}(x, \hbar)(16)$, имеет элементы

$$
\begin{aligned}
\sigma_{x x}\left(\Psi_{\nu}\right) & =\frac{\hbar}{4}\left[C_{0} D_{\nu} C_{0}^{+}+C_{0}^{*} D_{\nu} C_{0}^{\mathrm{T}}\right], \\
\sigma_{p p}\left(\Psi_{\nu}\right) & =\frac{\hbar}{4}\left[B_{0} D_{\nu} B_{0}^{+}+B_{0}^{*} D_{\nu} B_{0}^{\mathrm{T}}\right], \\
\sigma_{p x}\left(\Psi_{\nu}\right) & =\frac{\hbar}{4}\left[B_{0} D_{\nu} C_{0}^{+}+B_{0}^{*} D_{\nu} C_{0}^{\mathrm{T}}\right] .
\end{aligned}
$$

Здесь $D_{\nu}$ - диагональная матрица, $D_{\nu}=\left\|\left(2 \nu_{j}+1\right) \delta_{k j}\right\|_{n \times n}$. Тогда согласно общим конструкциям ковариантного подхода [16] решение задачи (5), (6) с $\left.\Psi\right|_{t=0}=\Psi_{\nu}(x, \hbar)$ из (16) на интервале $[0, T]$ представляется в следующем виде:

$$
\begin{aligned}
\Psi_{\nu}(x, t)= & N_{\nu} \exp \left[\frac{i}{\hbar} S_{\varkappa}(t, \hbar)\right] \exp \left[\frac{i}{\hbar}\langle P(t, \hbar), x-X(t, \hbar)\rangle\right] \times \\
& \times \exp \left[\left\langle x-X(t, \hbar), B C^{-1}(t)(x-X(t, \hbar)\rangle\right] \frac{1}{\sqrt{\operatorname{det} C(t)}}\left[\widehat{\Lambda}^{+}(t)\right]^{\nu} 1 .\right.
\end{aligned}
$$

Здесь вещественная классическая часть фазы есть

$$
\begin{aligned}
S_{\varkappa}(t, \hbar)= & \int_{0}^{t}[\langle P, \dot{X}\rangle(\tau, \hbar)-H(P(\tau, \hbar), X(\tau, \hbar))] d \tau- \\
& \quad-\varkappa \int_{0}^{t}\left[V(z(\tau, \hbar), z(\tau, \hbar))+\frac{1}{2} \operatorname{Sp}\left(V_{w w}^{\prime \prime}(z(\tau, \hbar), z(\tau, \hbar)) \Delta(\tau, \hbar)\right)\right] d \tau,
\end{aligned}
$$

$Z(t, \hbar)=(P(t, \hbar), X(t, \hbar)) \in \mathbb{R}^{2 n}$, а вектор-оператор $\widehat{\Lambda}^{+}(t)$ определяется формулой (18) при замене матриц $B_{0}$ и $C_{0}$ на матрицы $B(t)$ и $C(t)$, соответственно. Остается описать преобразование $\left(B_{0}, C_{0}\right) \rightarrow(B(t), C(t))$. Оно возникает в процессе построения решения системы Гамильтона-Эренфеста (из уравнения относительно $\Delta$ в $(11))$ в виде регулярного ряда теории возмущений по параметру $\hbar \rightarrow 0$ с точностью до $O\left(\hbar^{3 / 2}\right)$. Дело в том, что для построения главного члена асимптотики $\left(\bmod \hbar^{3 / 2}\right)$ уравнения (5) в форме (20) вместо точного решения системы (11)-(13) достаточно использовать ее приближенное по $\hbar \rightarrow 0$ решение $z(t, \hbar)=z^{0}(t)+\hbar z^{1}(t)+O\left(\hbar^{3 / 2}\right)$ и $\Delta^{0}=\Delta^{0}(t, \hbar)$ - главный член разложения $\Delta(t, \hbar)$, имеющий, как мы отмечали во введении, порядок $O(\hbar)$ (см. также формулы $(19)$ для $\left.\Delta^{0}(0, \hbar)\right)$. Выпишем соответствующие уравнения для $z^{0}(t), z^{1}(t)$ и $\Delta^{0}(t, \hbar)$ :

$$
\begin{array}{rlrl}
\dot{z}^{0} & =J\left(H_{z}\left(z^{0}\right)+\varkappa V_{z}\left(z^{0}, z^{0}\right)\right), & & z^{0}(0)=\left(p_{0}, x_{0}\right), \\
\dot{z}^{1}=J M_{z}\left(z^{0}\right) z^{1}+F\left(z^{0}, \Delta^{0}\right), & & z^{1}(0)=0, \\
\dot{\Delta}^{0} & =J M_{\varkappa}\left(z^{0}\right) \Delta^{0}-\Delta^{0} M_{\varkappa}\left(z^{0}\right) J, & & \left.\Delta^{(0)}\right|_{t=0}=\Delta\left(\Psi_{\nu}\right),
\end{array}
$$


где $F(z, \Delta)=J \nabla_{z} \operatorname{Sp}\left[\left(H_{z z}(z)+\varkappa V_{z z}(z, z)+\varkappa V_{w w}(z, z)\right) \Delta\right] / 2 \hbar, M_{z}\left(z_{0}\right)=M_{\varkappa}\left(z_{0}\right)+$ $\varkappa V_{z w}\left(z_{0}, z_{0}\right)$.

УтВеРЖДЕНИЕ 1. Пусть $A(t)$ - матрица Коши системы

$$
\dot{A}=J M_{\varkappa}\left(z^{0}(t)\right) A(t), \quad A(0)=E_{2 n},
$$

где $E_{2 n}=\left\|\delta_{i j}\right\|_{2 n \times 2 n}$. Тогда решение задачи Коши (24) определяется формулой

$$
\Delta^{(0)}(t, \hbar)=A(t) \Delta\left(\Psi_{\nu}\right) A^{+}(t) .
$$

ДокАЗАТЕЛЬСтво утверждения производится прямыми вычислениями и является, по существу, отражением того факта, что уравнение (24) относительно матрицы $\Delta^{0} J$ есть известное уравнение Лакса в методе обратной задачи (см., например, [22]).

Гамильтонову систему (25) мы называем системой в вариациях с самодействием. Она является системой в вариациях в окрестности решения $z^{0}(t)$ в общепринятом значении лишь при $\varkappa=0$, т.е. в случае линейного уравнения Шредингера с символом гамильтониана $H(z)$. В нелинейной ситуации она играет ту же важную роль, что и обычная система в вариациях в линейной теории комплексного ростка Маслова. В частности, матрицы $B(t)$ и $C(t)$ в формуле (16) определяются как решение задачи Коши для системы в вариациях с самодействием:

$$
\left(\begin{array}{c}
\dot{B} \\
\dot{C}
\end{array}\right)=J M_{\varkappa}\left(z^{0}(t)\right)\left(\begin{array}{c}
B \\
C
\end{array}\right),\left.\quad B\right|_{t=0}=B_{0},\left.\quad C\right|_{t=0}=C_{0} .
$$

В силу того, что эта система является гамильтоновой, для матриц $B(t)$ и $C(t)$ выполняются в любой момент $t \in[0, T]$ те же свойства, что и для матриц $B_{0}, C_{0}$ B (15).

ЗАмечАниЕ. Очевидно, что матрица Коши $A(t)$ системы $(25)$ представима через решение задачи (27) в виде

$$
A(t)=\left(\begin{array}{ll}
B(t) & B^{*}(t) \\
C(t) & B^{*}(t)
\end{array}\right)\left(\begin{array}{ll}
B_{0} & B_{0}^{*} \\
C_{0} & B_{0}^{*}
\end{array}\right)^{-1} .
$$

\section{3. КВАНТОВАНИЕ УСТОЙЧИВЫХ ТОЧЕК ПОКОЯ СИСТЕМЫ ГАМИЛЬТОНА-ЭРЕНФЕСТА}

Найдем приближенные $\left(\bmod \hbar^{3 / 2}\right)$ точки покоя системы $(11)$, используя эквивалентную ей с точностью до $O\left(\hbar^{3 / 2}\right), \hbar \rightarrow 0$, систему уравнений $(22)-(24)$.

Пусть символы $H(z), V(z, w)$ в операторе $\widehat{H}_{\varkappa}(2)$ таковы, что уравнение нулевого приближения (22) имеет точку покоя, т.е. стационарное решение $z^{0}(t)=z_{*}^{0}=$ const, удовлетворяющее уравнению

$$
J\left(H_{z}\left(z_{*}^{0}\right)+V_{z}\left(z_{*}^{0}, z_{*}^{0}\right)\right)=0 .
$$

Будем также предполагать, что матрица системы в вариациях с самодействием, отвечающая этой точке $z_{*}^{0}$, является невырожденной,

$$
\operatorname{det} J M_{\varkappa}\left(z_{*}^{0}\right) \neq 0 \text {. }
$$

2 Теоретическая и математическая физика, т. 150, № 1, 2007 г. 
Существование стационарного решения системы $(24)$, соответствующего $z_{*}^{0}$, определяется следующей леммой.

Лемма. Стационарное решение $\Delta^{0}(t, \hbar)=\Delta_{*}=$ const уравнения (24) существует тогда, когда система в вариациях с самодействием (25) является устойчивой, т.е. постоянная матрица $J M_{\varkappa}\left(z_{*}^{0}\right)$ диагонализуется, и ее спектр лежит на мнимой оси (спектр $\exp \left[J M_{\varkappa}\left(z_{*}^{0}\right) t\right]$ лежит на единичной окружности).

ДокАЗАТЕЛЬСтво. Не ограничивая общности, будем считать, что все собственные числа матрицы $J M_{\varkappa}\left(z_{*}^{0}\right)$ простые. Обозначим их через $\pm i \Omega_{k}$, а через $f_{k}^{ \pm}=$ $\left(w_{k}^{ \pm}, z_{k}^{ \pm}\right)^{\mathrm{T}}$ - отвечающие им собственные векторы, нормированные условием $\left\langle f_{k}^{+}, J^{\mathrm{T}} f_{k}^{-}\right\rangle=2 i, k=1,2, \ldots, n$. Вектор-функции $a_{k}^{+}(t)=\exp \left[i \Omega_{k} t\right] f_{k}^{+}, \quad a_{k}^{-}(t)=$ $\exp \left[-i \Omega_{k} t\right] f_{k}^{-}, k=1,2, \ldots, n$, (решения Флоке) образуют симплектический базис в пространстве $\mathbb{C}^{2 n}$ системы $(25)$.

Обозначим $B\left(z_{*}^{0}\right)$ и $C\left(z_{*}^{0}\right)(n \times n)$-матрицы, составленные из "импульсных" $w_{k}^{+}$и "координатных" $z_{k}^{+}$компонент векторов $f_{k}^{+}, k=1,2, \ldots, n$. Тогда матрицы

$$
\begin{aligned}
& B(t)=B\left(z_{*}^{0}\right) \operatorname{diag}\left(\exp \left[i \Omega_{1} t\right], \ldots, \exp \left[i \Omega_{n} t\right]\right), \\
& C(t)=C\left(z_{*}^{0}\right) \operatorname{diag}\left(\exp \left[i \Omega_{1} t\right], \ldots, \exp \left[i \Omega_{n} t\right]\right)
\end{aligned}
$$

суть матричные решения задачи (27) с начальными данными $C_{0}=C\left(z_{*}^{0}\right), B_{0}=$ $B\left(z_{*}^{0}\right)$. Выберем эти матрицы в качестве матриц $B(t)$ и $C(t)$ в формуле $(27)$ для матрицы Коши системы $(25)$ и зададим начальную матрицу дисперсий $\Delta\left(\Psi_{\nu}\right)$ в $(26)$ с помощью формул (19), положив в них $B_{0}=B\left(z_{*}^{0}\right), C_{0}=C\left(z_{*}^{0}\right)$. Тогда прямой выкладкой устанавливается, что при таком выборе матрица $A(t) \Delta\left(\Psi_{\nu}\right) A^{+}(t)$ не зависит от времени и, следовательно, в силу формулы (26) определяет стационарное решение $\Delta^{0}(t, \hbar)=$ const $=\Delta_{*}$ уравнения $(24)$.

СлеДСТВИЕ. Стационарное решение уравнения (24), m.е. вещественная $(2 n \times$ 2n)-матрица $\Delta_{*}$, определяется формулами

$$
\begin{aligned}
\Delta_{*} & =\left(\begin{array}{cc}
\sigma_{p p}\left(z_{*}^{0}\right) & \sigma_{p x}\left(z_{*}^{0}\right) \\
\sigma_{x p}\left(z_{*}^{0}\right) & \sigma_{x x}\left(z_{*}^{0}\right)
\end{array}\right), \quad \sigma_{x p}=\sigma_{p x}^{\mathrm{T}}, \\
\sigma_{x x}\left(z_{*}^{0}\right) & =\frac{\hbar}{4}\left[C_{0}\left(z_{*}^{0}\right) D_{\nu} C_{0}^{+}\left(z_{*}^{0}\right)+C_{0}^{*}\left(z_{*}^{0}\right) D_{\nu} C_{0}^{\mathrm{T}}\left(z_{*}^{0}\right)\right], \\
\sigma_{p p}\left(z_{*}^{0}\right) & =\frac{\hbar}{4}\left[B_{0}\left(z_{*}^{0}\right) D_{\nu} B_{0}^{+}\left(z_{*}^{0}\right)+B_{0}^{*}\left(z_{*}^{0}\right) D_{\nu} B_{0}^{\mathrm{T}}\left(z_{*}^{0}\right)\right], \\
\sigma_{p x}\left(z_{*}^{0}\right) & =\frac{\hbar}{4}\left[B_{0}\left(z_{*}^{0}\right) D_{\nu} C_{0}^{+}\left(z_{*}^{0}\right)+B_{0}^{*}\left(z_{*}^{0}\right) D_{\nu} C_{0}^{\mathrm{T}}\left(z_{*}^{0}\right)\right] .
\end{aligned}
$$

Пусть точка покоя $z_{0}^{*}$ системы (29) устойчива в линейном приближении, т.е. собственные векторы $\tilde{f}_{k}$ и собственные значения $\widetilde{\Omega}_{k}$ матрицы $J M_{z}\left(z_{0}^{*}\right)$ удовлетворяют условиям леммы. Векторы $f_{k}^{ \pm}$образуют симплектический базис в $\mathbb{C}^{2 n}$. Отсюда, обратив матрицу $J M_{\varkappa}\left(z_{*}^{0}\right)$, мы без труда найдем первую поправку $z_{*}^{1}$ в $z$-компоненте точки покоя из уравнения (23). В результате найдем, что

$$
z_{*}^{1}=\operatorname{Re}\left(\sum_{k=1}^{n} \tilde{f}_{k}^{+} \widetilde{\Omega}_{k}^{-1}\left\langle F\left(z_{*}^{0}\right) \Delta_{*}, J \tilde{f}_{k}^{+}\right\rangle\right) .
$$

Таким образом мы доказали следующее 
УТВЕРЖДЕНИЕ 2. Пусть выполнены условия на символы $H(z), V(z, w)$, обеспечивающие существование устойчивого в линейном приближении решения $z_{*}^{0}$ уравнения (29), и пусть система в вариациях с самодействием (с невырожденной матрицей $\left.J M_{\varkappa}\left(z_{*}^{0}\right)\right)$ устойчива. Тогда приближенная $\left(\bmod \hbar^{3 / 2}\right)$ точка покоя $y^{*}=\left(z_{*}=z_{*}^{0}+\hbar z_{*}^{1}, \Delta_{*}\right)$ системь Гамильтона-Эренфеста определяется формулами (29), (34), (33).

Подставив теперь построенное стационарное решение $y^{*}$ и матрицы $B(t), C(t)$ из (31) в формулы предыдущего раздела, мы получаем траекторно-когерентное состояние $\Psi_{\nu}(x, t)$, циклически зависящее от времени. Действительно, в этом случае матрица $B C^{-1}(t)$ не зависит от времени и равна $B\left(z_{*}^{0}\right) C\left(z_{*}^{0}\right)^{-1}$. Вещественная часть фазы решения (21) является линейной функцией $t$,

$$
S_{\varkappa}(t, \hbar)=-t\left[H\left(z_{*}(\hbar)\right)+\varkappa\left(V\left(z_{*}(\hbar), z_{*}(\hbar)\right)\right)+\frac{1}{2} \operatorname{Sp}\left[V_{w w}\left(z_{*}(\hbar), z_{*}(\hbar)\right) \Delta_{*}\right]\right],
$$

а амплитуда задается формулой

$$
\frac{\left[\widehat{\Lambda}^{+}(t)\right]^{\nu} 1}{\sqrt{\operatorname{det} C(t)}}=\exp \left[-i t \sum_{k=1}^{n} \Omega_{k}\left(\nu_{k}+\frac{1}{2}\right)\right] \frac{\left[\widehat{\Lambda}^{+}(0)\right]^{\nu} 1}{\sqrt{\operatorname{det} C\left(z_{*}^{0}\right)}} .
$$

Определим последовательность чисел $E_{\nu}(\hbar)$ и последовательность функций $\Psi_{\nu}(x, \hbar)$, $\nu \in \mathbb{Z}_{+}^{n}$, формулами

$$
\begin{aligned}
E_{\nu}(\hbar)= & H\left(z_{*}(\hbar)\right)+\hbar \sum_{k=1}^{n} \Omega_{k}\left(\nu_{k}+\frac{1}{2}\right)+ \\
& +\varkappa\left(V\left(z_{*}(\hbar), z_{*}(\hbar)\right)+\frac{1}{2} \operatorname{Sp}\left[V_{w w}\left(z_{*}(\hbar), z_{*}(\hbar)\right) \Delta_{*}\right],\right. \\
\Psi_{\nu}(x, t)= & N_{\nu} \exp \left[\frac{i}{\hbar}\left\langle P_{*}(\hbar), x-X_{*}(\hbar)\right\rangle\right] \times \\
& \times \exp \left[\frac{i}{2 \hbar}\left\langle x-X_{*}(\hbar), B C^{-1}\left(z_{*}^{0}\right)\left(x-X_{*}(\hbar)\right\rangle\right] \frac{1}{\sqrt{\operatorname{det} C\left(z_{*}^{0}\right)}}\left[\widehat{\Lambda}^{+}(0)\right]^{\nu} 1,\right.
\end{aligned}
$$

где $z_{*}(\hbar)=z_{*}^{0}+\hbar z_{*}^{1}, \Delta_{*}$ - “устойчивая" $\left(\bmod \hbar^{3 / 2}\right)$ точка покоя системы ГамильтонаЭренфеста (11). Здесь $z_{*}(\hbar)=\left(P_{*}(\hbar), X_{*}(\hbar)\right)$, матрицы $B\left(z_{*}^{0}\right), C\left(z_{*}^{0}\right)$ определены выше, а оператор рождения $\widehat{\Lambda}^{+}(0)$ задан формулой $(18)$, в которой $B_{0}=B\left(z_{*}^{0}\right)$, $C_{0}=C\left(z_{*}^{0}\right), w_{j} \in \mathbb{C}^{n}, z_{j} \in \mathbb{C}^{n}-$ столбцы матриц $B\left(z_{*}^{0}\right), C\left(z_{*}^{0}\right)$, соответственно.

Таким образом, доказана теорема, представляющая основной результат работы.

ТеОрема. Пусть выполнены условия (14) на символь оператора типа Хартри $\widehat{H}_{\varkappa}(2)$ и условия утверждения 2. Тогда последовательность чисел $E_{\nu}(\hbar)(37) u$ функций $\Psi_{\nu}(x, \hbar)(38)$ является квазиклассической спектральной серией (с точностъю $\left.O\left(\hbar^{3 / 2}\right)\right)$ оператора $\widehat{H}_{\varkappa}$ типа Хартри (2), отвечающей точке покоя системы Гамильтона-Эренфеста, ассочиированной с оператором $\widehat{H}_{\varkappa}$. 


\section{4. ПРИМЕРЫ}

\section{1. Спектр осциллятора в постоянном магнитном поле с нелинейным} квадратичным потенциалом. Проиллюстрируем представленные выше конструкции уравнением типа Хартри с квадратичным потенциалом в нелинейной части и с гамильтонианом линейной части, который отвечает полю осциллятора, находящегося в постоянном магнитном поле:

$$
\begin{aligned}
\widehat{H} & =E \Psi, \quad \widehat{H}=\frac{1}{2 m}\left(\hat{p}-\frac{e}{c} A(x)\right)^{2}+\frac{k x^{2}}{2}, \\
\widehat{V}(\Psi) & =\frac{1}{2} \int_{\mathbb{R}^{3}} d y\left[a x^{2}+2 b\langle x, y\rangle+c y^{2}\right]|\Psi(y)|^{2}, \quad A(x)=\frac{1}{2}[H, x] .
\end{aligned}
$$

Здесь $a, b, c$ - параметры нелокального потенциала; $m, k$ - параметры системы; $H$ - напряженность внешнего магнитного поля, $[H, x]$ - векторное произведение, $H, x, y \in \mathbb{R}^{3}$.

Для квадратичного гамильтониана $\widehat{H}_{\varkappa}$ приближенная система $(22)-(24)$ совпадает с исходной системой Гамильтона-Эренфеста (11). Так как $F(z, \Delta)=0$, то уравнения (24), описывающие движение средних $z(t, h)$, интегрируются независимо от уравнения для вторых моментов $\Delta_{2}(t)$. Нетрудно заметить, что $z^{1}(t)=0$ и $z(t)=z^{0}(t), \Delta_{2}(t)=\Delta_{2}^{0}(t)$. Уравнение $(22)$ можно переписать как систему

$$
\begin{aligned}
& \dot{p}=-(k+\varkappa a) x-\frac{e}{2 m c}[H, p]-\frac{e^{2}}{4 m c^{2}}[H,[x, H]], \\
& \dot{x}=\frac{1}{m} p-\frac{e}{2 m c}[H, x] .
\end{aligned}
$$

Стационарное решение $z_{*}$ системы (40) имеет вид

$$
x_{*}=0, \quad p_{*}=0 .
$$

Решение системы Гамильтона-Эренфеста для вторых моментов получим с помощью решений системы в вариациях.

Решение системы в вариациях (27) можно представить в форме

$$
B(t)=i \mathrm{M}(t) \mathrm{BA}, \quad C(t)=\mathrm{M}(t) \mathrm{CA} .
$$

Здесь $\mathrm{M}(t)=\operatorname{diag}\left(e^{i \Omega_{0} t}, e^{i \Omega_{1} t}, e^{i \Omega_{2} t}\right)$,

$$
\begin{aligned}
& \mathrm{C}=\sqrt{\frac{1}{2 m}}\left(\begin{array}{ccc}
\sqrt{2 \Omega_{0}^{-1}} & 0 & 0 \\
0 & \sqrt{\omega_{a}^{-1}} & \sqrt{\omega_{a}^{-1}} \\
0 & i \sqrt{\omega_{a}^{-1}} & -i \sqrt{\omega_{a}^{-1}}
\end{array}\right), \quad \mathrm{B}=\sqrt{\frac{m}{2}}\left(\begin{array}{ccc}
\sqrt{2 \Omega_{0}} & 0 & 0 \\
0 & i \sqrt{\omega_{a}} & i \sqrt{\omega_{a}} \\
0 & -\sqrt{\omega_{a}} & \sqrt{\omega_{a}}
\end{array}\right), \\
& \mathrm{A}=\left(e_{n}, e_{\varphi}, e_{\theta}\right)=\left(\begin{array}{ccc}
\cos \varphi \sin \theta & \sin \varphi & \cos \varphi \cos \theta \\
\sin \varphi \sin \theta & -\cos \varphi & \sin \varphi \cos \theta \\
\cos \theta & 0 & -\sin \theta
\end{array}\right) \\
& \Omega_{0}=\sqrt{\frac{k+\varkappa a}{m}}, \quad \Omega_{1}=-\frac{\omega_{c}}{2}+\omega_{a}, \quad \Omega_{2}=\frac{\omega_{c}}{2}+\omega_{a}, \\
& \omega_{c}=\frac{e|H|}{2 m c}, \quad \omega_{a}=\sqrt{\omega_{c}^{2}+\Omega_{0}^{2}} .
\end{aligned}
$$


Единичный вектор $e_{n}$ определяет направление магнитного поля, а совокупность векторов $\left\{e_{n}, e_{\varphi}, e_{\theta}\right\}$ образует ортонормированный базис в $\mathbb{R}^{3}$.

Для решения данной задачи нам достаточно знать не всю матрицу $\Delta_{2}$, а только ее блок дисперсии координат. Подставив (41) в (33), имеем

$$
\sum_{k=1}^{3} \sigma_{x_{k} x_{k}}\left(z_{*}^{0}\right)=\left(\nu_{0}+\frac{1}{2}\right) \frac{\hbar}{m \Omega_{0}}+\left(\nu_{1}+\frac{1}{2}\right) \frac{\hbar}{m \omega_{a}}+\left(\nu_{2}+\frac{1}{2}\right) \frac{\hbar}{m \omega_{a}} .
$$

Следовательно, квазиклассические $\left(\bmod h^{3 / 2}\right)$ собственные функции оператора типа Хартри имеют вид

$$
\Psi_{\nu}(x, \hbar)=\left(\frac{1}{\sqrt{2}}\right)^{|\nu|} \frac{1}{\sqrt{\nu !}} \frac{m^{3 / 4} \Omega_{0}^{1 / 4} \omega_{a}^{1 / 2}}{(\pi \hbar)^{3 / 4}} \exp \left\{\frac{i}{\hbar}\left(\frac{1}{2}\langle\chi, Q \chi\rangle\right)\right\} H_{\nu}(\xi) .
$$

Здесь $Q=\operatorname{diag}\left(i m \Omega_{0}, i m \omega_{a}, i m \omega_{a}\right), \nu=\left(\nu_{0}, \nu_{1}, \nu_{2}\right)$ - мультииндекс, $\chi=\mathrm{A}^{\mathrm{T}} x, H_{\nu}(\xi)-$ многомерные полиномы Эрмита, порожденные матрицей $W$,

$$
H_{\nu}(\xi)=(-1)^{|\nu|}\left(\frac{\partial}{\partial \xi}-2 W \xi\right)^{\nu} \cdot 1, \quad \xi=-\frac{i}{\sqrt{\hbar}} \mathrm{C}^{-1} \chi, \quad W=\mathrm{C}^{+}\left(\mathrm{C}^{-1}\right)^{\mathrm{T}}
$$

Собственные значения, отвечающие функциям (43), определяются выражением

$$
\begin{aligned}
E_{\nu}=\hbar & \left(\nu_{0}+\frac{1}{2}\right)\left(\frac{\varkappa c}{2 m \Omega_{0}}+\Omega_{0}\right)+\hbar\left(\nu_{1}+\frac{1}{2}\right)\left(\frac{\varkappa c}{2 m \omega_{a}}+\Omega_{1}\right)+ \\
& +\hbar\left(\nu_{2}+\frac{1}{2}\right)\left(\frac{\varkappa c}{2 m \omega_{a}}+\Omega_{2}\right) .
\end{aligned}
$$

Решение спектральной задачи (40) при $k=0, H=0$ с потенциалом взаимодействия гауссова типа дано в [23].

4.2. Одномерный осциллятор. Выберем линейный гамильтониан и нелокальное взаимодействие в виде

$$
\begin{aligned}
\widehat{H} & =\frac{\mu \hat{p}^{2}}{2}+\frac{\sigma x^{2}}{2}+\frac{\rho(x \hat{p}+\hat{p} x)}{2}, \\
\widehat{V}(\Psi) & =\frac{1}{2} \int_{-\infty}^{+\infty} d y\left[a x^{2}+2 b x y+c y^{2}\right]|\Psi(y)|^{2} .
\end{aligned}
$$

Здесь $a, b, c$ - параметры нелокального потенциала; $\mu, \sigma, \rho$ - параметры системы; $x, y \in \mathbb{R}^{1}$.

Система Гамильтона-Эренфеста для моментов первого порядка примет вид

$$
\begin{aligned}
& \dot{p}=-\sigma_{0} x-\rho p, \\
& \dot{x}=\mu p+\rho x,
\end{aligned}
$$

а для моментов второго порядка запишется как

$$
\begin{aligned}
& \dot{\sigma}_{x x}=2 \mu \sigma_{x p}+2 \rho \sigma_{x x}, \\
& \dot{\sigma}_{x p}=\mu \sigma_{p p}-\tilde{\sigma} \sigma_{x x}, \\
& \dot{\sigma}_{p p}=-2 \rho \sigma_{p p}-2 \tilde{\sigma} \sigma_{x p},
\end{aligned}
$$


где

$$
\sigma_{0}=\sigma+\varkappa(a+b), \quad \tilde{\sigma}=\sigma+\varkappa a
$$

Положим

$$
\widetilde{\Omega}=\sqrt{\sigma_{0} \mu-\rho^{2}}, \quad \Omega=\sqrt{\tilde{\sigma} \mu-\rho^{2}} .
$$

Будем считать, что

$$
\widetilde{\Omega}^{2}=\sigma_{0} \mu-\rho^{2}>0, \quad \Omega^{2}=\tilde{\sigma} \mu-\rho^{2}>0 .
$$

Общее решение системы (46) в этом случае дается выражениями

$$
\begin{aligned}
& X(t)=C_{1} \sin \widetilde{\Omega} t+C_{2} \cos \widetilde{\Omega} t \\
& P(t)=\frac{1}{\mu}\left(\widetilde{\Omega} C_{1}-\rho C_{2}\right) \cos \widetilde{\Omega} t-\frac{1}{\mu}\left(\widetilde{\Omega} C_{2}+\rho C_{1}\right) \sin \widetilde{\Omega} t .
\end{aligned}
$$

Соответственно для системы (47) имеем

$$
\begin{aligned}
\sigma_{x x}(t)= & C_{3} \sin 2 \Omega t+C_{4} \cos 2 \Omega t+C_{5}, \\
\sigma_{x p}(t)= & \frac{1}{\mu}\left(\Omega C_{3}-\rho C_{4}\right) \cos 2 \Omega t-\frac{1}{\mu}\left(\Omega C_{4}+\rho C_{3}\right) \sin 2 \Omega t-\frac{\rho}{\mu} C_{5}, \\
\sigma_{p p}(t)= & \frac{1}{\mu^{2}}\left(\left(\rho^{2}-\Omega^{2}\right) C_{3}+2 \rho \Omega C_{4}\right) \sin 2 \Omega t+ \\
& \quad+\frac{1}{\mu^{2}}\left(\left(\rho^{2}-\Omega^{2}\right) C_{4}-2 \rho \Omega C_{3}\right) \cos 2 \Omega t+\frac{\tilde{\sigma}}{\mu} C_{5} .
\end{aligned}
$$

Здесь $C_{l}, l=1,2, \ldots, 5,-$ произвольные постоянные.

Для решения спектральной задачи нам требуется стационарное решение системы Гамильтона-Эренфеста (46), (47), которое с учетом (33) получается при следующем выборе параметров:

$$
\mathfrak{C}_{1}=\mathfrak{C}_{2}=\mathfrak{C}_{3}=\mathfrak{C}_{4}=0, \quad \mathfrak{C}_{5}=\frac{\hbar \mu}{\Omega}\left(\nu+\frac{1}{2}\right) .
$$

Решение системы в вариациях (27) можно представить в виде

$$
B(t)=e^{i \Omega t} \frac{-\rho+i \Omega}{\sqrt{\Omega \mu}}, \quad C(t)=e^{i \Omega t} \sqrt{\frac{\mu}{\Omega}} .
$$

Следовательно, собственные функции оператора Хартри имеют вид

$$
\psi_{\nu}(x)=\frac{i^{\nu}}{\sqrt{\nu !}}\left(\frac{1}{\sqrt{2}}\right)^{\nu}\left(\frac{1}{\pi \hbar}\right)^{1 / 4}\left(\frac{\Omega}{\mu}\right)^{1 / 4} \exp \left\{-\frac{i}{2 \hbar} \frac{\rho}{\mu} x^{2}-\frac{1}{2 \hbar} \frac{\Omega}{\mu} x^{2}\right\} H_{\nu}\left(\sqrt{\frac{\Omega}{\hbar \mu}} x\right)
$$

где $H_{\nu}(\zeta)$ - полиномы Эрмита. Отвечающие им собственные значения определяются выражением

$$
E_{\nu}=\hbar\left(\nu+\frac{1}{2}\right)\left(\frac{\varkappa c \mu}{2 \Omega}+\Omega\right)
$$

Решение спектральной задачи (45) при $\rho=0$ приведено в [14]. 
Благодарности. Авторы признательны М. Ф. Кондратьевой, внимательно прочитавшей рукопись и сделавшей ряд ценных замечаний. Работа частично поддержана Программой поддержки ведущих научных школ (грант № НШ-5103.2006.2), РФФИ (гранты № 05-01-000968, НCM 05-01-22002) и DFG; Ф. Н. Литвинец поддержан стипендией некоммерческого фонда “Династия" в рамках МЦФФ в Москве.

\section{Список литературы}

[1] М. В. Карасев, В. П. Маслов, Нелинейные скобки Пуассона. Геометрия и квантование, Наука, М., 1991.

[2] С. Р. де Гроот, Л. Г. Сатторп, Электродинамика, Мир, М., 1982.

[3] Y. Lai, H. A. Haus, Phys. Rev. A, 40 (1989), 844-853; 854-866.

[4] Л. П. Питаевский, УФН, 168 (1998), 641-653.

[5] А.С. Давыдов, Солитоны в молекулярных системах, Наукова думка, Киев, 1984.

[6] M. Born, Z. Phys., 38 (1926), 803-827; П. Эренфест, "Замечание о приближенной справедливости классической механики в рамках квантовой механики", Относительность. Квантъь. Статистика, Наука, М., 1972, 82-84.

[7] В.П. Маслов, Теория возмущений и асимптотические методы, МГУ, М., 1965; В. П. Маслов, М.В. Федорюк, Квазиклассическое приближение для уравнений квантовой механики, Наука, М., 1976.

[8] В.П. Маслов, Асимптотические методы и теория возмущений, Наука, М., 1988.

[9] В. П. Маслов, Операторные методы, Наука, М., 1973.

[10] В.П. Маслов, Комплексный метод ВКБ в нелинейных уравнениях, Наука, М., 1977; В. В. Белов, С. Ю. Доброхотов, ТМФ, 92:2 (1992), 215-254.

[11] М. В. Карасев, А. В. Перескоков, ТМФ, 79:2 (1989), 198-208; 97:1 (1993), 78; Изв. РАН. Сер. Матем., 65:3 (2001), 33-72; 65:6 (2001), 57-98; М. В. Карасев, В. П. Маслов, "Алгебры с общими перестановочными соотношениями и их приложения. II. Операторные унитарно-нелинейные уравнения”, Итоги науки и техники. Сер. современные проблемы математики, 13, ВИНИТИ, М., 1979, 145-267; M. V. Karasev, V. P. Maslov, J. Soviet Math., 15 (1981), 273-368.

[12] V. V. Belov, A. Yu. Trifonov, A. V. Shapovalov, Int. J. Math. Math. Sci., 32:6 (2002), 325370.

[13] В. В. Белов, А. Ю. Трифонов, А. В. Шаповалов, ТМФ, 130:3 (2002), 460-492.

[14] A. L. Lisok, A. Yu. Trifonov, A. V. Shapovalov, J. Phys. A, 37 (2004), 4535-4556; А. Л. Лисок, А. Ю. Трифонов, А. В. Шаповалов, ТМФ, 141:2 (2004), 228-242.

[15] A. L. Lisok, A. Yu. Trifonov, A. V. Shapovalov, Proc. Inst. Math. NAS Ukr., 50:3 (2004), 1454-1465; Symm., Integr. Geom.: Methods and Appl. (SIGMA), 1 (2005), 007; F. N. Litvinets, A. V. Shapovalov, A. Yu. Trifonov, J. Phys. A, 39 (2006), 1191-1206.

[16] V. G. Bagrov, V. V. Belov, A. Yu. Trifonov, Ann. Phys. (NY), 246:2 (1996), 231-280; В. Г. Багров, В.В. Белов, А. Ю. Трифонов, "Квазиклассически сосредоточенные состояния уравнения Шредингера", Летняя школа “Волга-7'95", VII Петровские чтения (Казань, Россия, 22 июня - 2 июля 1995 г.), ред. А.В. Аминова, Изд-во БОГ, Казань, 1996, 15-136.

[17] В. В. Белов, М.Ф. Кондратьева, Матем. заметки, 56:6 (1994), 27-39; 58:6 (1995), 803-817.

[18] В. Г. Багров, В. В. Белов, М.Ф. Кодратьева, ТМФ, 98:1 (1994), 48-55; V. G. Bagrov, V. V. Belov, M. F. Kondratyeva, et al., J. Moscow Phys. Soc., 3 (1993), 309-320; "The quasiclassical localization of the states and a new approach of quasi-classical approximation in quantum mechanics", Particle Physics, Gauge Fields and Astrophysics, Accademia Nazilonale dei Lincei, Rome, 1994, 132-142; V. G. Bagrov, V. V. Belov, A. Yu. Trifonov, "New methods for semiclassical approximation in quantum mechanics", Proc. 
Int. Workshop Quantum Systems: New Trends and Methods (Minsk, May 23-29, 1994), eds. A. O. Barut, I. D. Feranchuk, Ya. M. Shnir, L. M. Tomil'chik, World Scientific, Singapore, 1995, 533-543.

[19] М.А. Малкин, В.И. Манько, Динамические симметрии и когерентные состояния квантовых систем, Наука, М., 1979; А. М. Переломов, Обобщенные когерентные состояния и их применение, Наука, М., 1987.

[20] В. Г. Багров, В. В. Белов, И. М. Тернов, ТМФ, 50:3 (1982), 390-396; V. G. Bagrov, V.V. Belov, I. M. Ternov, J. Math. Phys., 24:12 (1983), 2855-2859; В. В. Белов, В. П. Маслов, ДАН СССР, 305:3 (1989), 574-580; 311:4 (1990), 849-854; V. G. Bagrov, V.V. Belov, A. Yu. Trifonov, Semiclassical trajectory-coherent approximation in quantum mechanics: II. High order corrections to the Dirac operators in external electromagnetic field, quant-ph/9806017; В. В. Белов, М.Ф. Кондратьева, ТМФ, 92:1 (1992), 41-60; V.G. Bagrov, V.V. Belov, A. Yu. Trifonov, A. A. Yevseyevich, Class. Quant. Grav., 8 (1991), 515-527; 1349-1359; 1833-1846; V. G. Bagrov, A. Yu. Trifonov, A. A. Yevseyevich, Class. Quant. Grav., 9 (1992), 533-543.

[21] Г. Бейтмен, А. Эрдейи, Высшие трансцендентные функиии. Функиии Бесселя. Функиии параболического иилиндра. Ортогональные многочлены, Наука, М., 1966.

[22] В.Е. Захаров, С.В. Манаков, С.П. Новиков, Л.П. Питаевский, Теория солитонов: Метод обратной задачи, Наука, М., 1980; М. Абловиц, Х. Сегур, Солитоны и метод обратной задачи, Мир, М., 1983.

[23] И. В. Сименог, ТМФ, 30:3 (1977), 408-414.

Поступила в редакцию 26.05.2006 